\title{
单层膜包裹的金纳米粒子的表面状态对铁氰根离 子和硫代硫酸根离子间的氧化还原反应的影响 ${ }^{*}$
}

\author{
李 迪 ${ }^{\circledR}$ 孙春燕 ${ }^{\circledR}$ 黄云杰 ${ }^{\circledR}$ 李景虹 ${ }^{(2 * *}$ 陈少伟 ${ }^{\circledR}$
}

(1)电分析化学国家重点实验室 中国科学院长春应用化学研究所, 长春 130022; (2)清华大学化学系, 北京 100084;

(3)Department of Chemistry, University of California, Santa Cruz, Santa Cruz, CA 95064, USA)

\begin{abstract}
摘要利用三维自组装膜包覆的金纳米粒子为电子转移媒介体, 研究电子在三维自组装膜表 面的转移行为. 文中以 3-颈基丙酸和 11-巯基十一酸包覆的金纳米粒子为催化剂, 催化铁氰化钾 与硫代硫酸钠之间的反应. 该催化反应的机理为金纳米粒子作为电子转移的媒介体，电子在金纳 米粒子表面转移的速度决定着反应的速度. 因此, 电子在这种三维自组装膜上的转移速度和反应 速度成正比, 而该反应的反应速度可以由铁氰化钾的紫外光谱的变化得到. 实验中得到的表观电 子转移速度比理论计算隧道电流产生的电子转移速度的最大值小几个数量级, 这可能是由于纳 米粒子能级不连续性造成的.
\end{abstract}

\section{关键词电子转移 三维自组装膜 电子媒介体}

由于纳米粒子特有的物理, 化学, 电子以及磁学 性质, 有关纳米粒子的研究正成为科学的热点 ${ }^{[1]}$. 对 于催化电子转移反应，纳米粒子有广泛的应用前景 $[2]$. 粒子材料，尺寸，表面官能团等结构参数对其催化行 为有显著的影响．例如，尽管大尺寸的金并没有催化 活性，近年来的研究发现金纳米粒子对 $\mathrm{CO}$ 氧化为 $\mathrm{CO}_{2}$ 有明显的催化活性, 同时仅仅改变粒子的大小 ${ }^{[3]}$ 和表面荷电状态 ${ }^{[4]}$ 就可以明显的增强催化活性. 在相 关的研究中, 钟川健 ${ }^{[5,6]}$ 等人发现即使在金纳米粒子 表面包裹一层表面活性剂单层膜后，金纳米粒子对
甲醇和CO的电化学氧化仍有明显的催化活性. 其他 的研究表明, 对于一些重要的有机反应(如加氢或水 合)，利用树枝状化合物 ${ }^{[79]}$ 或聚合物 ${ }^{[10]}$ 包覆的Pt，Pd 纳米粒子作为催化剂, 催化活性有明显的提高.

本文中，我们试图利用Brust反应 ${ }^{[11]}$ 制得的金纳 米粒子来研究纳米粒子表面三维自组装膜上的电子 转移行为. 铁氧化钾与硫代硫酸钠之间的氧化还原 反应是一个经典的贵金属团簇催化反应. 研究表明, 该催化反应是表面控制的 ${ }^{[12 ~ 14]}$. 即金属团簇作为高 度分散的电子媒介体，在铁氧化钾和硫代硫酸钠之 
间传递电子. 本文采用不同链长的硫醇自组装膜(3颈基丙酸和 11-颈基十一酸)包裹的金纳米粒子为催 化剂催化上述氧化还原反应, 研究纳米粒子, 特别是 纳米粒子的表面状态对这一反应的影响. 计算了这 种三维自组装膜上的表观电子转移速度常数并与二 维自组装膜进行了比较.

\section{1 实验部分}

\section{1 试剂}

3-颈基丙酸(MPA), 11-颈基十一酸(MUA), 牛血 清白蛋白 (BSA, 分子量约为 66,000) 购自 Aldrich. $\mathrm{K}_{3}\left[\mathrm{Fe}(\mathrm{CN})_{6}\right], \mathrm{Na}_{2} \mathrm{~S}_{2} \mathrm{O}_{3}, \mathrm{HAuCl}_{4} \cdot 3 \mathrm{H}_{2} \mathrm{O}, \mathrm{NaBH}_{4}$, 丙三醇 为分析醇. 实验用水经过 Milli-Q $\left(18.3 \mathrm{M} \Omega \cdot \mathrm{cm}^{-1}\right)$ 蒸馏水系统处理.

\subsection{Au-MPCs 及其 BSA 包覆物的合成}

在 MPA 和 MUA 存 在 时, 利用 $\mathrm{NaBH}_{4}$ 还原 $\mathrm{HAuCl}_{4} \cdot 3 \mathrm{H}_{2} \mathrm{O}$ 可以得到 MPA和 MUA包裹的金纳米粒 子(MPA monolayer protected $\mathrm{Au}$ clusters and MUA monolayer protected Au clusters, 简称为 Au-MPAMPCs和 Au-MUA-MPCs). Au-MPA- MPCs 是在水相 中合成的, 而 Au-MUA-MPCs 是在水和乙醇(体积比 为 $1: 1$ )的混合溶剂中合成的. 为了进一步增加保护层 的厚度, 降低电子通过保护层转移的速度, 在MPA和 MUA层的外部进一步引入了 BSA. 由于过量的 $\mathrm{NaBH}_{4}$ 并未从体系中除去, 所以溶液的 $\mathrm{pH}$ 为碱性. 在 静电作用下, BSA 吸附在 Au-MPA-MPCs 和 Au-MUA-MPCs 表面, 形成BSA 包覆物 (缩写为 Au-MPA@BSA-MPCs 和 Au-MUA@BSA-MPCs).

\section{3 实验装置}

$\mathrm{Fe}(\mathrm{CN})_{6}{ }^{3-}$ 的紫外吸收光谱是在Cary-100 (VARIAN, USA)紫外-可见光光谱仪上测定的. 在 $420 \mathrm{~nm}$ 处，铁氰根离子和金纳米粒子都有强烈的吸收. 在 $470 \mathrm{~nm}$ 处, 只有金纳米粒子有吸收, 此处的吸光系数 与 $420 \mathrm{~nm}$ 处比较接近 ${ }^{[12]}$. 因此, 对于未催化的反应, 铁氰根离子的吸光度是在 $420 \mathrm{~nm}$ 处记录的. 而金纳 米粒子催化剂存在时, 铁氰根离子的吸光度为 420 $\mathrm{nm}$ 处的峰强减去 $470 \mathrm{~nm}$ 处的峰强. 实验中, 将 $2 \mathrm{~mL}$
金纳米粒子和 $0.2 \mathrm{~mL}$ 铁氰化钾 $(10 \mathrm{mmol} / \mathrm{L})$ 加入到 1 $\mathrm{cm}$ 的石英比色㿼中, 然后加入 $0.2 \mathrm{~mL}$ 硫代硫酸钠 $(100 \mathrm{mmol} / \mathrm{L})$ 开始反应. Au-MPA-MPCs催化时, 紫 外光谱仪中的空白样品为水, 而Au-MUA-MPCs催化 时, 采用的空白样品为水和乙醇的混合溶剂(体积比 为 $1: 1$ ). 利用铁氰化钾的浓度 (由紫外吸光度得 到) 随时间的变化作图，所得直线的斜率即为本反应速 度的绝对值.

金纳米粒子的电镜图像由 JEOL-JEM-2010 (JEOL, 日本)透射电子显微镜得到, 操作电压为 200 $\mathrm{kV}$. 样品(约 $4 \mathrm{mg} / \mathrm{mL}$ ) 滴加在碳膜(厚度为 200 300 $\AA$ ) 覆盖的铜网上(230 目).

\section{2 结果与讨论}

\section{$2.1 \mathrm{Fe}(\mathrm{CN})_{6}{ }^{3-}$ 和 $\mathrm{S}_{2} \mathrm{O}_{3}{ }^{2-}$ 之间的反应途径}

$$
2 \mathrm{~S}_{2} \mathrm{O}_{3}{ }^{2-}+2\left[\mathrm{Fe}(\mathrm{CN})_{6}\right]^{3-} \rightarrow \mathrm{S}_{4} \mathrm{O}_{6}{ }^{2-}+2\left[\mathrm{Fe}(\mathrm{CN})_{6}\right]^{4-} \text { (1) }
$$

铁氭化钾与硫代硫酸钠之间的反应是经典的金 属胶体催化的氧化还原反应. 通常认为其催化机理 是表面控制, 即反应速度是由铁氧根离子和硫代 硫酸根离子在金属胶体表面交换电子的速度决定 的 ${ }^{[12 ~ 14]}$.

图 1(a)是反应进行 $3 \min$ 时铁氰化钾(初始浓度为 $2 \mathrm{mmol} / \mathrm{L})$ 的紫外吸收光谱. 内插图为铁氰化钾浓度 随时间变化的曲线, 曲线斜率 $\left(2.5 \times 10^{-4} \mathrm{mmol} / \mathrm{min}\right)$ 即 为此初始浓度下的反应速度. 图 1(b)为铁氰化钾的初 始浓度与反应速度之间的关系，线形关系表明本反 应对铁氰化钾为一级反应.

此外, 我们发现增加 $\mathrm{H}^{+}$的浓度会明显加快该反 应的速度(图 2(a)). (b)为反应速度和 $\left[\mathrm{H}^{+}\right]$之间的关系. 可以看到, 当 $\left[\mathrm{H}^{+}\right]$从 $2 \mathrm{mM}$ 增加到 $10 \mathrm{mmol} / \mathrm{L}$ 时, 反应 速度随之加快了大约 3 倍, 表明铁氰根离子和硫代硫 酸根离子之间初始反应是酸催化的 ${ }^{[15]}$. 这可以解释 为 $\left[\mathrm{H}^{+}\right]$的增加降低了两种反应物离子之间的静电排 斥作用，增加了它们之间碰撞的几率.

\section{2 金纳米颗粒在催化反应中的作用}

图 3 是Au-MPA@BSA-MPCs 和 Au-MUA@ BSA-MPCs 的电子显微镜照片. 当BSA包裹在金纳 米粒子表面时, 可以看到明显的各向异性特点 $[16]$, 即 

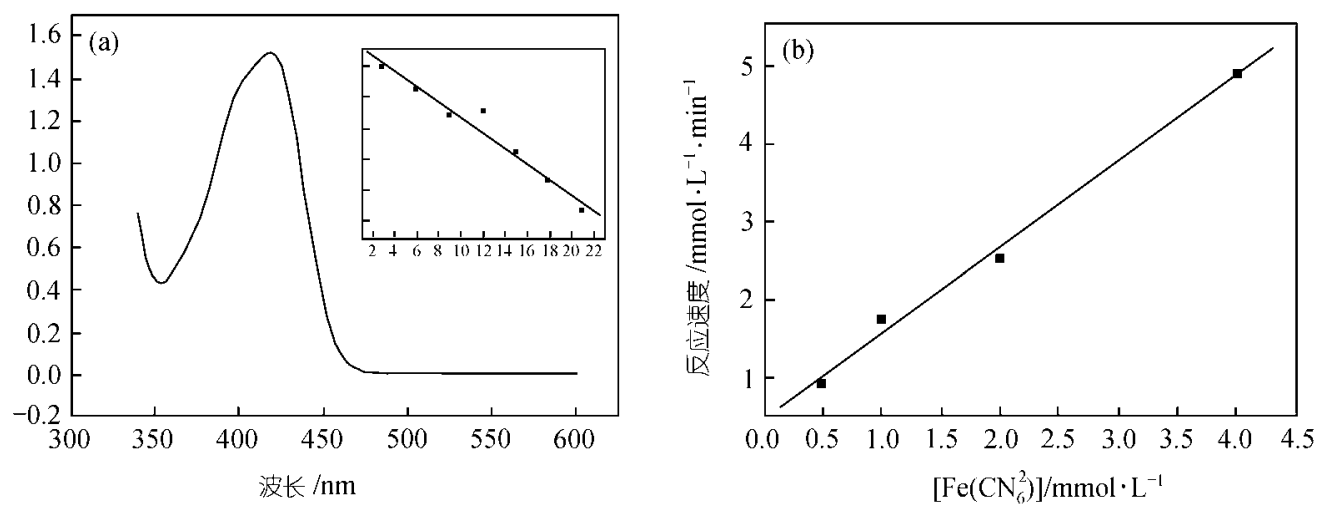

图 1

(a) 铁氭化钾 $(2 \mathrm{mmol} / \mathrm{L})$ 和硫代硫酸钠 $(100 \mathrm{mmol} / \mathrm{L})$ 反应 $3 \mathrm{~min}$ 时的紫外吸收光谱. 内插图为反应中铁氧化钾浓度随时间变化的曲线, 曲线斜率为 此条件时的反应速度; (b) 铁氰化钾的初始浓度与反应速度的关系曲线. 线性相关系数为 0.9964
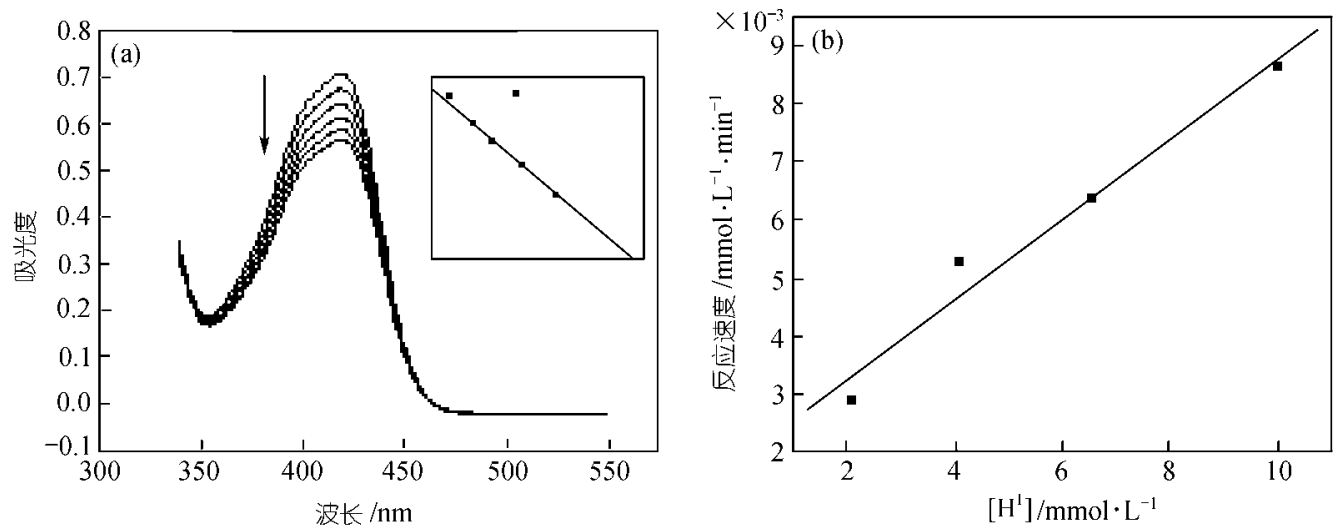

图 2

(a) 铁氭化钾(初始浓度 $10 \mathrm{mmol} / \mathrm{L}$ ), 硫代硫酸钠(初始浓度 $100 \mathrm{mmol} / \mathrm{L}$ ), $\mathrm{H}^{+}(4.1 \mathrm{mmol} / \mathrm{L}$ )时, 反应不同时间(从 5 30 min, 时间间隔为 $5 \mathrm{~min}$ )时的紫 外光谱. 内插图为反应中铁氰化钾浓度随时间变化的曲线, 曲线斜率为此条件时的反应速度; (b) $\mathrm{H}^{+}$的初始浓度与反应速度的关系. 线性相关系数 为 0.9841

金纳米粒子排成了链状，而没有BSA包覆时，却没有 出现这种现象. 本实验中制得的金纳米颗粒的粒径 大约为 $4 \mathrm{~nm}, 2 \mathrm{~mL}$ (浓度为 $0.28 \mathrm{mmol} / \mathrm{L}$ ) 溶液中纳米 粒子的总表面积为 $4.22 \mathrm{~m}^{2}$ (具体计算过程见附录), 该表面积远远大于传统的平面电极. X射线光电子能 谱(XPS)的结果表明纳米粒子中金原子的结合能为 $83.9 \mathrm{eV}$, 对应着零价的金.

为了验证本体系中金纳米粒子是否起电子转移 媒介体的作用, 我们采用了两种不同链长的硫醇, 11統基十一酸和 3-颈基丙酸, 作为配体稳定金纳米粒 子. 此外, 我们还引入了 BSA 来增加保护层的厚度 使电子转移速度减慢(反应速度如表 1 所示), 这样表 面状态对反应动力学的影响就更加明显. 如果本催
化反应确实是由表面电子转移反应控制的, 那么增 加纳米粒子表面保护层的厚度实际上就是增加了电 子由溶液进入金核的距离, 减慢了反应速度.

当Au-MUA-MPCs和Au-MUA@BSA-MPCs 被 用作催化剂时, 反应速度由 $2.90 \times 10^{-4} \mathrm{mmol} / \mathrm{L} \cdot \mathrm{min}^{-1}$ (Au-MUA-MPCs) 降 低到了 $1.32 \times 10^{-4}$ $\mathrm{mmol} / \mathrm{L} \cdot \mathrm{min}^{-1}(\mathrm{Au}-\mathrm{MUA} @ B S A-M P C s)$, 我们认为原 因可能是这样的, MUA的链比MPA长的多, 所以吸附 在Au-MUA-MPCs上的BSA层对电子转移的屏蔽作用 不如对Au-MPA-MPCs明显.

总之，本部分紫外光谱的实验表明 $\mathrm{Au}-\mathrm{MPA}-\mathrm{MPCs}$ 催化的反应速度 $\left(7.92 \times 10^{-3} \mathrm{~m}\right.$ $\left.\mathrm{mol} / \mathrm{L} \cdot \mathrm{min}^{-1}\right)$ 比Au-MUA-MPCs $\left(2.90 \times 10^{-4}\right.$ 

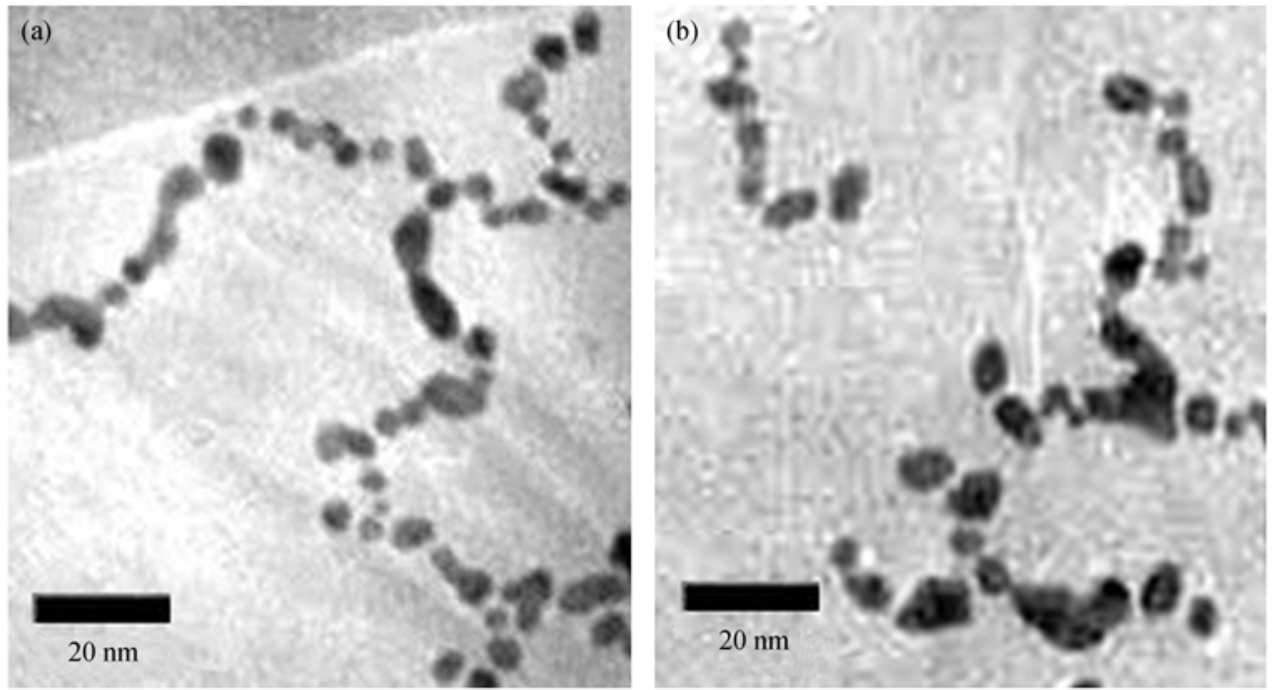

图 3 Au-MPA@BSA-MPCs(左)和 Au-MUA@BSA-MPCs(右)的 TEM 照片

表 1 使用不同催化剂得到的催化反应速度 ${ }^{a)}$

\begin{tabular}{lcccc}
\hline \multicolumn{1}{c}{ 催化剂 } & Au-MPA-MPCs & Au-MPA@BSA-MPCs & Au-MUA-MPCs & Au-MUA@BSA-MPCs \\
\hline 反应速度 $\left(\times 10^{4}, \mathrm{mmol} / \mathrm{L} \cdot \mathrm{min}^{-1}\right)$ & 79.2 & 29.3 & 2.9 & 1.32 \\
相关系数 & 0.99 & 0.98 & 0.99 & 0.97 \\
\hline
\end{tabular}

a)反应速度通过检测催化剂存在时铁氰化钾紫外吸收峰强度的变化得到

$\left.\mathrm{mmol} / \mathrm{L} \cdot \mathrm{min}^{-1}\right)$ 大的多. 用BSA将金纳米粒子包裹起 来之后，反应速度降低. 但Au-MUA@BSA-MPCs体 系中反应速度的降低没有Au-MPA@BSA-MPCs体系 明显. 图 4 给出了本催化反应的机理. 电子给体 $\mathrm{S}_{2} \mathrm{O}_{3}{ }^{2}$ 给出一个电子, 这个电子通过BSA和羒酸保护 层转移给金核，然后金核将这个电子再通过表面的 保护层转移给 $\mathrm{Fe}(\mathrm{CN})_{6}{ }^{3-}$, 而这一步可以通过检测 $\mathrm{Fe}(\mathrm{CN})_{6}{ }^{3-}$ 紫外吸收峰强度的变化得到.

\section{3 电子在 BSA 包覆的三维自组装膜上的转移速}

度

表观电子转移速度 $\left(K_{a p p}\right)$ 可以通过(2)式计算得到

$$
K_{a p p}=\frac{i_{c}}{n F A c_{0}},
$$

其中 $i_{c}$ 为电流强度, $F$ 是法拉第常数, $A$ 为电极的总表 面积, $c_{0}$ 是探针分子的浓度, $n$ 为反应中包括的电子数. 本实验中, $A$ 为金纳米粒子的总表面积. $i_{C}$ 可以通过(3) 式得到

$$
i_{c}=F \times v,
$$

其中 $v$ 是金纳米粒子催化的反应速度. 由此计算得到

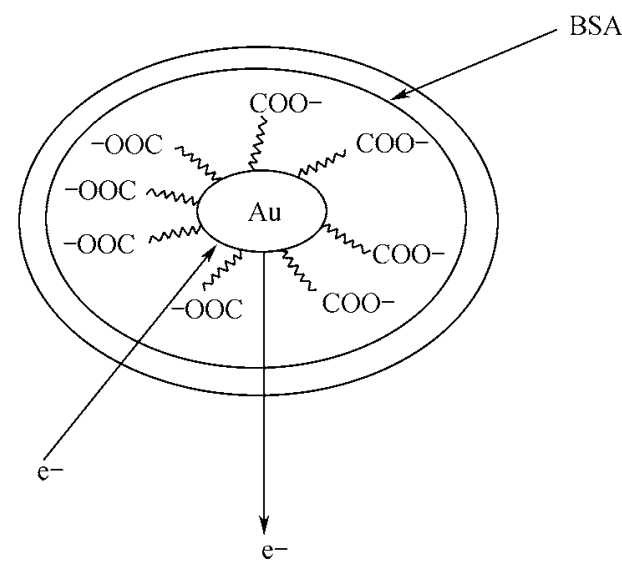

图 4 电子在金纳米粒子表面转移示意图

本实验中通过 MPA 和 MUA 保护层的 $K_{a p p}$ 分别为 $3.75 \times 10^{-6}$ 和 $1.37 \times 10^{-7} \mathrm{~cm} / \mathrm{s}$.

如果通过这种三维自组装膜的电子转移是以通 过针孔或缺陷的质量传递的电流为主, 那么改变溶 液的黏度就会改变电流的大小. 然而本实验中采用 甘油和水的混合溶剂(体积比为 $1:$ 1)来改变溶剂的 黏度，并没有发现反应速度有明显改变. 因此可以确 
认本实验中的电子转移是以隧穿电流为主的. 利用 Marcus理论计算得到的 $\mathrm{Fe}(\mathrm{CN})_{6}{ }^{3-}$ 在二维自组装膜上 的最大 $K_{a p p}$ 是 $2.3 \times 10^{-3} \mathrm{~cm} / \mathrm{s}$, 比本实验中得到的在三 维自组装膜上的数据大得多 117$]$.

Marcus 理论指出致密自组装膜的电子转移是由 隧穿电流控制的, 电子转移速度与给体和受体之间 的能带重叠程度成正比. 电极的能带密度通常当作 常数放入指数前因子中. 然而在本实验中电极 (金纳 米粒子)的体积非常小, 此时能级是不连续的, 因此 能带密度不能当成常数. 这可能是本实验得到的电 子转移速度很小的原因.

\section{3 结论}

在本文中, 我们研究了电子在三维自组装膜上 的转移行为. 采用 Au-MPA-MPCs 和 Au-MUA-MPCs 催化铁氰化钾和硫代硫酸钠之间的反应. 催化机理 可以认为金纳米粒子作为氧化还原反应中的电子转 移媒介体. 因此电子在保护层中的转移速度和反应 速度呈正比. 计算得到的表观电子转移速度比 Marcus 理论预测的铁氰化钾通过二维自组装膜的最 大值小几个数量级.

\section{参考文献}

1 Roucoux A, Schulz J, Patin H. Reduced transition metal colloids: A novel family of reusable catalysts? Chem Rev, 2002, 102(10): 3757 3778[DOI]

2 Adams D M, Brus L, Chidsey C E D, et al. Charge transfer on the nanoscale: Current status. J Phys Chem B, 2003, 107(28): 6668 6697[DOI]

3 Choudhary T V, Goodman D W. Oxidation catalysis by supported gold Nano-clusters. Top Catal, 2002, 21(1-3): 25 34

$4 \mathrm{Fu} \mathrm{Q}$, Saltsburg H. Flytzani-Stephanopoulos M. Active nonmetallic Au and Pt species on Ceria-Based water-gas shift catalysts. Science, 2003, 301(5635): 935 938[DOI]

5 Luo J, Jones V W, Maye M M,et al. Thermal activation of molecularly-wired gold nanoparticles on a substrate as catalyst. J Am Chem Soc, 2002, 124(47): 13988 13989[DOI]

6 Maye M M, Lou Y B, Zhong C J. Core-shell gold nanoparticle assembly as novel electrocatalyst of CO oxidation. Langmuir, 2000, 16(19): 7520 7523[DOI]

7 Niu Y H,Yeung L K, Crooks R M. Size-selective hydrogenation of olefins by dendrimer-encapsulated palladium nanoparticles. J Am Chem Soc, 2001, 123(18): 6840 6846[DOI]
8 Scott R W J, Datye A K, Crooks R M. Bimetallic palladium-platinum dendrimer-encapsulated catalysts. J Am Chem Soc, 2002, 125(13): 3708 3709

9 Crooks R M, Zhao M Q, Sun L, et al. Dendrimer-encapsulated metal nanoparticles: Synthesis, characterization, and applications to catalysis. Acc Chem Res, 2001, 34(3): 181 190[DOI]

10 Narayanan R, El-Sayed M A. Effect of catalysis on the stability of metallic nanoparticles: Suzuki reaction catalyzed by PVP-Palladium nanoparticles. J Am Chem Soc, 2003, 125(27): 8340 8347[DOI]

11 Brust M, Walker M, Bethell D, et al. Synthesis of thiol-derivatised gold nanoparticles in a two-phase liquid-liquid system. J Chem Soc, Chem Commun, 1994, 801 802

12 Li Y, Petroski J, El-Sayed M A. Activation energy of the reaction between hexacyanoferrate(III) and thiosulfate ions catalyzed by platinum nanoparticles. J Phys Chem B, 2000, 104(47): 10956 10959[DOI]

13 Freund P L, Spiro M. Colloidal catalysis: The effect of sol size and concentration. J Phys Chem, 1985, 89 (7): 1074 1077

14 Freund P L, Spiro M. Catalysis by colloid gold of the reaction between ferricyanide and thiosulphate ions. J Chem Soc, Faraday Trans 1, 1986, 82: 2277 2282

15 Howlett K E, Wedzicha B L. Kinetics of the reaction between hexacyanoferrate (III) and thiosulfate ions: The initial reaction. Inorgan. Chim Acta, 1976, 18: 133 138

16 Zhang F X, Li H. Israel L B, Daras J G, et al. Colorimetric detection of thiol-containing amino acids using gold nanoparticles. Analyst, 2002, 127(4): 462 465[DOI]

17 Finklea H O. Electroanalytical Chemistry. Bard A J, Rubinstein I,ed. Marcel Dekker: New York, 1996.Vol 19, 109 335

附录

由 TEM 照片可知,Au-MPCs 的粒径为 $4 \mathrm{~nm}$. 因此将 Au-MPCs 视为球体, 以 $4 \mathrm{~nm}$ 的金球的质量除以一个金原 子的质量, 就可以得到一个 Au-MPCs中所包含的金原子数 ( $m$ ). 本实验中 $m$ 为 2000. 由 UV-vis 谱图知 Au-MPCs 的摩 尔浓度 $(c)$ 约为 $0.28 \mathrm{mmol} / \mathrm{L}$. 因此溶液中 $\mathrm{Au}-\mathrm{MPCs}$ 的数量 (n)可以通过下公式计算得到:

$$
n=\frac{c \times N}{m},
$$

计算所得的 $n$ 为 $8.4 \times 10^{16}$. 因此本文中 $A u-M P C s(A)$ 的总面 积为 $A=n \times 4 \pi r^{2}=4.22 \mathrm{~m}^{2}$. 